\title{
The influence of early neutrophil-Leishmania interactions on the host immune response to infection
}

\author{
Flavia L. Ribeiro-Gomes and David Sacks* \\ Laboratory of Parasitic Diseases, National Institute of Allergy and Infectious Diseases, National Institutes of Health, Bethesda, MD, USA
}

\section{Edited by:}

Stephen M. Beverley, Washington

University in St. Louis, USA

Reviewed by:

Jordi Torrelles, Ohio State

University, USA

Michael L. Vasil, University of

Colorado Medical School, USA

*Correspondence:

David Sacks, Laboratory of Parasitic

Diseases, National Institute of

Allergy and Infectious Diseases,

National Institutes of Health,

4 Center Dr. MSC 0425, Bethesda,

MD 20892-0425, USA.

e-mail:dsacks@nih.gov
Neutrophils are the first cells recruited to the dermal site of Leishmania infection following injection by needle or sand fly bite. The role of neutrophils in either promoting or suppressing host immunity remains controversial. We discuss the events driving neutrophil recruitment, their interaction with the parasite and apoptotic fate, and the nature of their encounters with other innate cells. We suggest that the influence of the neutrophil response on infection outcome critically depends on the timing of their recruitment and the tissue environment in which it occurs.

Keywords: neutrophils, Leishmania, inflammation, immune response, sand fly, apoptosis, dendritic cells, macrophages
Neutrophils are readily mobilized innate immune cells and essential for host defense against a variety of pathogens (Kobayashi and DeLeo, 2009). Patients with severe neutropenia or chronic granulomatous disease (CGD) suffer a wide range of microbial infections (Vento and Cainelli, 2003; Segal, 2005). Neutrophils are also recruited during acute sterile inflammation where they contribute to tissue repair (Kim et al., 2006; McDonald et al., 2010). Given their potent arsenal of constituents used for host defense, including reactive oxygen species (ROS), antimicrobial peptides, and serine proteases, the resolution of inflammation requires that dying neutrophils are removed from the inflammatory site by other phagocytic cells, such as macrophages and dendritic cells (DCs), to avoid their uncontrolled release of injurious contents (Segal, 2005). It is not surprising that any imbalance in the recruitment and removal of these cells is associated with numerous pathologies, including chronic infections and inflammatory disorders (Kono and Rock, 2008).

Leishmaniasis is a vector-borne disease caused by the intracellular protozoan Leishmania. There are an estimated 2 million new cases annually (1.5 million cases of cutaneous leishmaniasis and 500,000 cases of visceral leishmaniasis), with about 12 million people currently infected (www.who.int/leishmaniasis/burden). For each Leishmania species studied, neutrophils have been found recruited to the infection site following the delivery of parasites by sand fly bite or by needle injection (Beil et al., 1992; TacchiniCottier et al., 2000; Peters et al., 2008; Thalhofer et al., 2011; Ribeiro-Gomes et al., 2012). Numerous in vitro and in vivo studies have been carried out to understand the role of neutrophils in host defense against Leishmaniasis (Tacchini-Cottier et al., 2000; Ribeiro-Gomes et al., 2004, 2007; Peters et al., 2008, 2009; Charmoy et al., 2010a,b; Filardy et al., 2010). Because of the heterogeneity of these studies in terms of host species and mouse strain (human, dog, susceptible, or resistant mice), the tissue source and site of recruitment (blood, peritoneum, skin), and the time frame of the recruitment (hours, days, weeks) the impact of the neutrophilic response on promoting host resistance or susceptibility to Leishmania infection remains a matter of some debate. In keeping with the theme of this volume, this review will focus on the early events driving neutrophil recruitment, and their fate and immunologic consequences following Leishmania infection initiated by the bite of an infected sand fly or needle inoculation in the skin.

\section{RECRUITMENT}

The first observations concerning the early recruitment of neutrophils to the site of Leishmania infection were made by Wilson et al. (1987), who described a mixed neutrophil and monocytic infiltrate within $1 \mathrm{~h}$ following intra-dermal inoculation of L. donovani promastigotes in hamsters, and by Pompeu et al. (1991) and Beil et al. (1992), who described the predominant neutrophilic infiltrate within $3 \mathrm{~h}$ following high dose subcutaneous inoculation of Leishmania amazonensis or Leishmania major promastigotes, respectively, into the footpad of BALB/c or $\mathrm{C} 57 \mathrm{Bl} / 6$ mice. Studies by the group of Laskay confirmed that neutrophils were the first leukocyte population migrating to the site of L. major infection in the footpad (Muller et al., 2001). These findings have more recently been extended to the ear dermal inoculation site employing L. major (Peters et al., 2008; Charmoy et al., 2010a,b; Ribeiro-Gomes et al., 2012) or L. infantum (Thalhofer et al., 2011). A recent paper suggests that the kinetics of myeloid cell recruitment to the L. major injection site might be tissue specific, as neutrophil recruitment into the peritoneal cavity following i.p. injection of L. major was preceded at $30 \mathrm{~min}$ by GR-1 ${ }^{+}$monocytes (Goncalves et al., 2011). Our own studies used two-photon intra-vital microscopy (2P-IVM) to confirm that neutrophils are the first cells to infiltrate (within $30 \mathrm{~min}$.) 
the L. major loaded dermis of C57Bl/6 mice (Peters et al., 2008). Using 2P-IVM and flow cytometry, we furthermore provided the first description of the acute inflammatory response to sand fly transmitted infections, observing a rapid and sustained neutrophilic infiltrate at localized sand fly bite sites.

Neutrophil infiltration appears to be a multifactorial event. The wound repair and sterilization response triggered as a result of the tissue damage caused by the sand fly bite or by the needle injection is sufficient to drive the early recruitment of neutrophils. Following needle injection, the recruitment was comparable in sham injected mice over the first hour, confirming that the infiltrate was induced, at least in part, by the tissue injury. At subsequent time points, however, the recruitment was dependent on the infectious status of the inoculum (Ribeiro-Gomes et al., 2012). Indeed, Leishmania promastigotes produce a neutrophil chemotactic factor (LCF) and induce IL- 8 secretion by human neutrophils, a process that might amplify neutrophil recruitment (van Zandbergen et al., 2002). In the mouse, while CXCL1, a functional homologue of IL-8, has been shown to be rapidly upregulated in L. major infected skin (Muller et al., 2001), only complement C3 has been directly shown to be involved in early neutrophil recruitment (Jacobs et al., 2005). Importantly, neutrophil recruitment to the sand fly bite site is even more massive, localized, and sustained compared to the needle injected dermis, and the response elicited by infected or uninfected bites is comparable (Peters et al., 2008). Thus, the wound repair response to sand fly bites, in conjunction with the response induced by saliva components or microbiota introduced into the skin by the sand fly, might override the contribution of any parasite-derived signals on neutrophil recruitment. Relevant to this point, components of Lutzomyia intermedia and Lutzomyia longipalpis salivary glands were demonstrated to induce a rapid influx of neutrophils in BALB/c mice (Teixeira et al., 2005; de Moura et al., 2010).

The early influx of neutrophils to the site of infection in the skin is transient, with numbers returning to steady levels by 3 days following needle injection of L. major or L. infantum (Peters et al., 2009; Thalhofer et al., 2011). Resolution of the neutrophilic response to infected sand fly bites was also observed after 3 days, although cells were found persisting or still accumulating in localized bite sites as late as 8 days after transmission. Interestingly, a second wave of neutrophil recruitment, starting approximately 1 week after L. major infection in $\mathrm{C} 57 \mathrm{Bl} / 6$ mice, and coincident with the appearance of the lesion, was observed following either needle or sand fly challenge. It seems likely that the signals for recruitment of the first and subsequent waves of neutrophils to the site will be quite distinct, with mediators arising from the adaptive immune response contributing to the neutrophilic component of the granuloma and the evolution of the lesion. Interestingly, studies in IL-17 $-/-$ BALB/c mice demonstrated the role of IL-17 on neutrophil recruitment in later phase of infection (Lopez Kostka et al., 2009). The possible differences in the function and immunologic consequences of these early and later neutrophil populations will be considered below.

\section{FATE}

This section will consider the fate of both the parasite and the host cell following the interaction of neutrophils with Leishmania in vivo and in vitro. Amongst the earliest evidence that neutrophils can phagocytose Leishmania in vivo, Deane and Guimarges (1938) noted L. donovani amastigotes in smears of bone-marrow cells from a patient with kala-azar, not only in mononuclear cells, but also in eosinophils and neutrophils. Experimentally, parasitized neutrophils and macrophages were observed histologically during the first 2-3 days following high dose s.c. footpad injection of L. amazonensis promastigotes in BALB/c mice (Andrade et al., 1984; Pompeu et al., 1991), and primarily within neutrophils at $6 \mathrm{~h}$ following footpad injection of L. major in both C57Bl/6 and BALB/c mice (Lima et al., 1998). The rapid and efficient uptake of $L$. major promastigotes by inflammatory neutrophils was also observed when subcutaneous air pouches were used to establish an acute inflammatory infiltrate in BALB/c mice (Laufs et al., 2002). Wilson et al. were the first to carry out a histologic study of a strictly dermal injection site, and observed neutrophils as the predominant parasitized cell at $1 \mathrm{~h}$ following injection of $L$. donovani promastigotes (Wilson et al., 1987). More recently, using fluorescent labeled parasites and flow cytometry analysis, neutrophils were observed to be the predominant infected cells in the mouse ear dermis over the first few hours following injection of either L. major or L. infantum (Peters et al., 2008; Thalhofer et al., 2011; Ribeiro-Gomes et al., 2012).

The fate of ingested parasites is a key issue that remains to be clearly resolved. Early in vitro studies involving human neutrophils and $L$. donovani amastigotes or promastigotes reported killing that was attributable to the oxygen metabolites generated by the phagocytosis-induced respiratory burst (Chang, 1981; Pearson and Steigbigel, 1981). In vivo, ultrastructural studies of experimental lesions demonstrated the presence of degraded parasites inside neutrophil although these observations did not pertain to the early infiltrating cells (Andrade et al., 1984; Beil et al., 1992). Indirect evidence for early killing by neutrophils was the large number of lysed parasites observed in the extracellular space in the vicinity of neutrophils and leukocyte debris (Pompeu et al., 1991).

By contrast, there is conclusive evidence for the survival of L. major metacyclic promastigotes following their rapid uptake by neutrophils in the skin (Peters et al., 2008). By employing LYSeGFP mice infected with RFP-labeled L. major metacyclic promastigotes, a homogeneous population of infected neutrophils could be purified by cell sorting, and the number of viable organisms determined by plating the neutrophils on promastigote growth medium. Virtually, all of the infected neutrophils recovered from the ear dermis after one day were found to harbor at least one viable parasite, and when injected back into the ear dermis of naïve mice, the infected neutrophils produced the same course of lesion development as an identical number of metacyclic promastigotes injected directly. Intact parasites were also observed inside neutrophils in Giemsa stained inflammatory exudate cells recovered from air pouches 1 day after infection with L. major (Laufs et al., 2002). In vitro, L. donovani promastigotes were found to survive in mouse and dog neutrophils that was associated with their trafficking to an ERlike, lysosome-independent compartment (Gueirard et al., 2008). L. major and L. donovani promastigotes were also found to survive in human neutrophils that in this case was linked to the 
absence of a phagocytosis-induced respiratory burst, and prevention of early fusion of tertiary and specific granules with the parasite-containing phagosome (Mollinedo et al., 2010).

Intracellular survival following capture by neutrophils would be consistent with the expression of promastigote-derived molecules, including the cell surface lipophosphoglycan (LPG) and tartrate-resistant acid phosphatase, that have been shown to inhibit lysosome fusion and/or the respiratory burst and super oxide anion production in mouse or human neutrophils (Remaley et al., 1984; al Tuwaijri et al., 1990; Gueirard et al., 2008). Another molecule released by L. major promastigotes, the Leishmania lipid chemotactic factor (LCF), previously shown to induce recruitment of human neutrophils (van Zandbergen et al., 2002), was also shown to increase survival in neutrophils by interacting with the lipoxin A4 receptor (ALX) and providing a deactivating signal to these cells (Wenzel et al., 2009). As each of these molecules might undergo developmental stage modifications, already well described for LPG (Sacks, 1992), then it becomes especially critical to examine early Leishmanianeutrophils interactions using purified metacyclic stage promastigotes that are the infectious forms deposited by sand fly bite. The killing observed in some studies, particularly those that used poorly defined cultured promastigotes, might be explained by the absence or deficit in the number of suitably differentiated metacyclic stage parasites in the inocula. The trafficking of the parasite to an ER-like, non-degradative compartment was in fact shown to be far more efficient when purified metacyclics or stationaryphase as compared to logarithmic phase promastigotes were used (Gueirard et al., 2008).

The fate of the neutrophils themselves will directly impact the fate of the parasites that they harbor. Neutrophils are short-lived cells, however, their longevity might be modulated by various inflammatory stimuli and pathogens. Chemokines, growth factors or pattern recognition signals delay the apoptotic death program of neutrophils. Neutrophil apoptosis can also be delayed or accelerated following uptake of microorganisms. Leishmania infection of human blood or mouse peritoneal cavity derived neutrophils was shown to delay neutrophil apoptosis in vitro (Aga et al., 2002; van Zandbergen et al., 2004; Charmoy et al., 2010a,b). By contrast, ex vivo analyzes of neutrophils recovered from the L. major loaded dermis found that the uptake of L. major metacyclic promastigotes accelerated their apoptosis, as evidenced by their enhanced expression of PtdSer relative to the uninfected neutrophils recovered from the dermal site (Ribeiro-Gomes et al., 2012). Interestingly, sand fly vector saliva was also found to accelerate the apoptosis of murine inflammatory peritoneal neutrophils (Prates et al., 2011), though the apoptotic program of neutrophils recruited to sand fly $v s$. needle injection sites have yet to be compared. Surface exposure of PtdSer is one of a number of molecules marking apoptotic cells for recognition and removal by macrophages and DCs, and infected neutrophils injected back into the ear dermis of naïve mice were more efficiently captured by dermal DCs compared to uninfected cells (Ribeiro-Gomes et al., 2012). Macrophages have also been observed to phagocytose infected neutrophils in vitro which appear to deliver viable organisms to the macrophage (van Zandbergen et al., 2004; Gueirard et al., 2008), and is the basis for the "Trojan horse" infection model, discussed in more detail below. By contrast to the results involving DCs, however, the uptake of infected neutrophils by macrophages in the skin was not observed in vivo, either by 2P-IVM or flow cytometry. Instead, these studies concluded that most of the infected, apoptotic neutrophils lytically released viable parasites that were subsequently taken up by other phagocytes, including macrophages. The possibility that the released parasites are transitional forms better adapted to intra-macrophage survival has yet to be addressed. And even if infected neutrophils do not directly transfer their parasites to macrophages, the clearance of apoptotic bodies by macrophages within the inflammatory site would still be expected to deactivate these cells and promote the intra-survival of any Leishmania that they might also engulf.

Finally, the possibility that upon their recruitment to the site of infection, neutrophils are activated to die and release DNA and certain granular contents to form extracellular traps or nets, needs to be considered based mainly on in vitro studies involving human neutrophils and Leishmania promastigotes (Guimaraes-Costa et al., 2009; Gabriel et al., 2010). There was no consensus as to the fate of the entrapped parasites in these studies, with histone-dependent killing observed in the former, and LPG dependent survival described in the later. Whether any neutrophils the acute infiltrate actually die by NETosis has yet to be demonstrated.

\section{IMMUNOLOGIC CONSEQUENCES}

Neutrophils might markedly modulate the development of the anti-Leishmania immune response by their ability to secrete cytokines, chemokines and granular contents, and by their direct interaction with others inflammatory and resident cells at the site of infection (Tacchini-Cottier et al., 2000; Ribeiro-Gomes et al., 2004; Charmoy et al., 2007; Ribeiro-Gomes et al., 2007, 2012). Based on the fact that neutrophils rapidly undergo apoptosis, a number of studies have addressed the interaction of neutrophils, infected or not, with other innate immune cells in the context of apoptotic cell clearance by phagocytes.

In murine studies, co-culture of apoptotic, uninfected neutrophils with macrophages infected with Leishmania in vitro was shown to have different outcomes on parasite growth depending on the Leishmania species and the genetic background of the host. Engulfment of neutrophils by infected macrophages from resistant C57BL/6 mice reduced the parasite load that was dependent on secretion of TNF that appeared to overcome the deactivation signals initiated by apoptotic cell removal. The activation was also dependent on the expression of neutrophil elastase (NE), and on macrophage expression of TLR4 (Ribeiro-Gomes et al., 2004, 2007). Enhanced activation and killing were also observed using L. major infected, IFN $\gamma$-primed bone-marrow derived macrophages (BMDM) following their engulfment of $\mathrm{C} 57 \mathrm{Bl} / 6$ neutrophils (Filardy et al., 2010). By contrast, engulfment of dead syngeneic neutrophils by infected macrophages from susceptible $\mathrm{BALB} / \mathrm{c}$ mice exacerbated $L$. major growth through production of PGE2 and TGF- $\beta$ (Ribeiro-Gomes et al., 2004). The deactivating effect involving neutrophils and infected macrophages from $\mathrm{BALB} / \mathrm{c}$ mice may be Leishmania species restricted, however, since inflammatory neutrophils induced killing of L. amazonensis via 
NE and TNF in macrophages from both susceptible BALB/c and resistant $\mathrm{C} 3 \mathrm{H} / \mathrm{HePas}$ mice (de Souza Carmo et al., 2010), and $\mathrm{BALB} / \mathrm{c}$ neutrophils increased killing of $L$. braziliensis in infected macrophages through up-regulation of TNF and ROS (Novais et al., 2009). Thus for most in vitro studies, the engulfment of neutrophils by macrophages already infected with Leishmania was found to enhance killing, though it should be noted that in none of these studies was normal growth or survival of the strains in control cultures indicated, suggesting that the parasites used for infection were compromised from the outset.

Of greater concern is the relevance of these co-culture assays to the timing of neutrophil, macrophage, and parasite interactions in vivo. As detailed above, neutrophils are the first inflammatory cells to swarm into a needle or fly bite inoculation site in the skin, and they represent the vast majority of infected cells over the first hours of infection. Thus, for most macrophages in the inflammatory site, their apoptotic cell clearance functions will precede their direct encounter with the parasite. In this context, while primed, previously infected BMDM were more highly activated for killing following co-culture with apoptotic C57BL/6 neutrophils, they became more permissive to $L$. major growth if they were co-cultured with the neutrophils for 3 days prior to infection (Filardy et al., 2010). They also secreted high amounts of TNF, IL-10 and NO following challenge with LPS, a phenotype consistent with regulatory/M2b macrophages. These findings appear relevant to the phagocytosis of infected neutrophils by uninfected macrophages, central to the Trojan Horse infection model whereby apoptotic neutrophils are thought to silently deliver Leishmania to host macrophages in the absence of the activation signals induced via direct parasitization (Laskay et al., 2008). In support of this model, ingestion of L. major infected human neutrophils by autologous monocyte derived macrophages was observed in vitro that resulted in productive infections in the macrophages and their release of TGF- $\beta$ (van Zandbergen et al., 2004).

Based on the in vitro studies discussed above and the in vivo kinetic studies of the recruitment and infection of different myeloid cell populations in the L. major loaded dermis (RibeiroGomes et al., 2012), we propose that at early time points infected and non-infected neutrophils, or their apoptotic corpses, interact with and are taken up by non-infected macrophages, which can drive the development of IL-10 $10^{\text {hi }}$ IL-12 ${ }^{\text {low }}$ M2-like macrophages that are especially permissive to parasite survival and growth. The parasites might be acquired as per the Trojan horse model, or by capture of viable organisms released by dying neutrophils, a process better supported by our intra-vital imaging and flow cytometry studies (Peters et al., 2008; Ribeiro-Gomes et al., 2012).

There is a further complexity to the immune modulatory influence of neutrophils in the L. major loaded dermis, in so far as a second wave of neutrophil recruitment is observed starting 1 week post-infection, at a time when the intracellular infections have fully transitioned from neutrophils to macrophages, inflammatory monocytes and DCs (Ribeiro-Gomes et al., 2012). At this time point, neutrophils are more apt to encounter already infected macrophages, and their engulfment by these "primed" cells might activate them for greater killing, reflecting the timing and effects observed in the in vitro co-culture experiments described above.
The neutrophils present in these sequential waves of inflammation might themselves exert quite different effects on the immune response, given that the mediators conditioning their recruitment, activation, and death are apt to be quite distinct. Interestingly, in studies involving human resting neutrophils from blood, phagocytosis of apoptotic neutrophils by L. amazonensis infected macrophages induced an increase in the number of intracellular parasites, whereas phagocytosis of necrotic neutrophils induced killing of parasites via a mechanism dependent on TNF and NE (Afonso et al., 2008). It should be noted that resting blood human neutrophils, in contrast to inflammatory/activated neutrophils dying by necrosis, undergo apoptosis without release of their intracellular contents.

Finally, as referred to previously, the majority of infected DCs recovered from the skin one day after infection appeared to acquire their parasites via uptake of infected neutrophils (Ribeiro-Gomes et al., 2012). When used ex vivo to stimulate L. major primed $\mathrm{T}$ cells, these DCs performed poorly compared to infected, dermal DCs recovered from neutrophil depleted mice that presumably acquired their parasites directly. In studies employing bone-marrow derived DCs that had phagocytosed L. major infected neutrophils in vitro, their capacity to stimulate ovalbumin-specific CD8 ${ }^{+}$OT-I T cells to proliferate and release IFN- $\gamma$ was completely suppressed (Ribeiro-Gomes, unpublished). Thus, the in vitro findings provide ample evidence to suggest that the acute neutrophilic infiltrate elicited by needle injection and especially sand fly bite is exploited by Leishmania to impair the innate response of macrophages and DCs in order to establish infection and delay the onset of the cell-mediated immune response.

The role of neutrophils in promoting or suppressing the antileishmanial response in vivo, addressed primarily by treating mice with neutrophil depleting antibodies, remains a confusing issue (summarized in Table 1 ). The outcomes again vary depending on the parasite and mouse strains used, the tissue site of challenge, and the timing and specificity of the depleting antibody. The initial studies were carried out with the RB6$8 \mathrm{C} 5 \mathrm{mAb}$, which binds to the GR1 epitope present on Ly-6G, Ly6C, and Ly-6B.2 glycoproteins (Fleming et al., 1993). Neutrophils express high levels of Ly-6G and are efficiently depleted by RB6$8 \mathrm{C} 5$ treatment. However, it is now appreciated that other cells express GR1, including eosinophils, subpopulations of DCs and lymphocytes, inflammatory monocytes, and myeloid suppressor cells, and they are also recognized and depleted by RB6-8C5 (Tepper et al., 1992; Kusmartsev et al., 2000; Nakano et al., 2001; Geissmann et al., 2003; Matsuzaki et al., 2003). The epitope recognized by the NIMP-R $14 \mathrm{mAb}$ is not so clear, and although it has been used as a more neutrophil-specific depleting $\mathrm{Ab}$, our own analysis of blood $\mathrm{CD}_{1} 1 \mathrm{~b}^{+}$cells suggest that not only $\mathrm{Ly}_{6 \mathrm{G}}{ }^{+} \mathrm{Ly} 6 \mathrm{C}^{\text {int }}$ neutrophils, but also $\mathrm{Ly} 6 \mathrm{G}^{-} \mathrm{Ly} 6 \mathrm{C}^{\text {hi }}$ inflammatory monocytes are depleted $48 \mathrm{~h}$ after treatment (RibeiroGomes, unpublished). More recently, a new neutrophil depleting $\mathrm{Ab}, 1 \mathrm{~A} 8$, has been described (Daley et al., 2008), that binds specifically to the Ly6G glycoprotein and depletes neutrophils in the blood, leaving monocytes unaffected. The neutrophil depletion achieved using $1 \mathrm{~A} 8$, however, is incomplete and/or does not last as long as the RB6-8C5 depletion, with residual 
Table 1 | Leishmania infection outcomes following neutrophil depletion in vivo.

\begin{tabular}{|c|c|c|c|c|c|}
\hline $\begin{array}{l}\text { Mouse } \\
\text { strain }\end{array}$ & $\begin{array}{l}\text { Leishmania } \\
\text { strain }\end{array}$ & $\begin{array}{l}\text { Site and route } \\
\text { of infection }\end{array}$ & Antibody & Outcome & References \\
\hline C57BL/6 & L. major & Footpad (sc) & RB6-8C5 & $\begin{array}{l}\text { Increase parasite load in dLN } 6 \mathrm{~h}, 24 \mathrm{~h} \text {, } \\
16 \mathrm{~d} \text {, and } 29 \mathrm{~d} \text { p.i. }\end{array}$ & Lima et al. (1998) \\
\hline C57BL/6 & L. major & Footpad (sc) & NIMP-R14 & $\begin{array}{l}\text { Increase parasite load in footpad } 35 d \\
\text { p.i.; no difference } 65 \mathrm{~d} \text { p.i. }\end{array}$ & Tacchini-Cottier et al. (2000) \\
\hline C57BL/6 & L. major & Footpad (sc) & RB6-8C5 & Increase parasite load in dLN 10 d p.i. & Ribeiro-Gomes et al. (2004) \\
\hline C57BL/6 & L. donovani & iv & RB6-8C5 & $\begin{array}{l}\text { Increase parasite load in spleen and } \\
\text { liver } 14 \mathrm{~d} \text { p.i. }\end{array}$ & Smelt et al. (2000) \\
\hline $\mathrm{BALB} / \mathrm{c}$ & L. major & Footpad (sc) & RB6-8C5 & $\begin{array}{l}\text { No difference in dLN } 24 \mathrm{~h}, 16 \mathrm{~d} \text {, and } \\
29 \mathrm{~d} \text { p.i. }\end{array}$ & Lima et al. (1998) \\
\hline $\mathrm{BALB} / \mathrm{c}$ & L. major & Footpad (sc) & NIMP-R14 & $\begin{array}{l}\text { Decrease parasite load in footpad } \\
35 \text { d p.i. }\end{array}$ & Tacchini-Cottier et al. (2000) \\
\hline $\mathrm{BALB} / \mathrm{C}$ & L. donovani & iv & RB6-8C5 & $\begin{array}{l}\text { Increase parasite load in spleen and } \\
\text { liver } 14 \mathrm{~d} \text { p.i. }\end{array}$ & Smelt et al. (2000) \\
\hline $\mathrm{BALB} / \mathrm{C}$ & L. donovani & iv & NIMP-R14 & $\begin{array}{l}\text { Increase parasite load in spleen and } \\
\text { bone-marrow } 14 \mathrm{~d} \text { p.i. }\end{array}$ & McFarlane et al. (2008) \\
\hline $\mathrm{BALB} / \mathrm{C}$ & L. braziliensis & Ear (id) & RB6-8C5 & $\begin{array}{l}\text { Increase parasite load in ear and dLN } \\
14 \mathrm{~d} \text { p.i. }\end{array}$ & Novais et al. (2009) \\
\hline $\mathrm{BALB} / \mathrm{C}$ & L. infantum & iv & RB6-8C5 & $\begin{array}{l}\text { Increase parasite load in spleen } \\
22 \mathrm{~d} \text { p.i. }\end{array}$ & Rousseau et al. (2001) \\
\hline $\mathrm{BALB} / \mathrm{c}$ & L. infantum & Ear (id) & RB6-8C5 & $\begin{array}{l}\text { Decrease parasite load in ear; increase } \\
\text { in dLN } 1 \mathrm{~d} \text { p.i. }\end{array}$ & Thalhofer et al. (2011) \\
\hline
\end{tabular}

sc, subcutaneous; iv, intravenous; id, intradermal; p.i., post-infection.

neutrophils still present in the L. major inoculation site in the ear at $24 \mathrm{~h}$ (Ribeiro-Gomes et al., 2012), and neutrophil numbers starting to increase in the blood after $48 \mathrm{~h}$ (Daley et al., 2008).

The first study by Lima et al. (1998), showed that treatment with neutrophil depleting Ab (RB6-8C5) increased parasite growth in the dLN following subcutaneous inoculation of L. major in the footpad of $\mathrm{C} 57 \mathrm{Bl} / 6$ mice. Using the same parasite strain and route of infection, but a different neutrophil depleting Ab (NIMP-R14 or RB6-8C5), Tacchini-Cottier et al. (2000) and Ribeiro-Gomes et al. (2004) observed the same enhancement of early parasite growth in the footpad or dLN, respectively. More recently, using a natural infection model involving L. major transmission by the bite of infected sand flies, Peters et al. (2008) observed substantially reduced parasite loads in the inoculation site at 1 and 4 weeks post infection in C57Bl/6 mice treated at the time of transmission with RB6-8C5. Neutrophil depletion using NIMP-R14 mAb shortly after sand fly challenge was also shown to enhance the protection conferred by a killed Leishmania vaccine (Peters et al., 2009). Neutrophil depletions using 1A8 or RB6-8C5 also facilitated early Leishmania specific $\mathrm{CD}^{+} \mathrm{T}$ cell priming in mice needle challenged with $L$. major in the ear dermis (Ribeiro-Gomes et al., 2012). It should be emphasized that in comparison to the needle injection site in the ear dermis, and especially the sand fly bite site in the ear, the number of neutrophils recruited to and taking up L. major in the sub-cutaneous needle injection site in the footpad is substantially less (Ribeiro-Gomes et al., unpublished), and presumably accounts for the differences in the immune response and infection outcomes following neutrophil depletion in these different settings. The experience with L. infantum infection models reinforces the importance of the inoculation site in defining the role that the neutrophilic response might play in infection outcome. Neutrophil depleted BALB/c mice were more susceptible to L. infantum when challenged i.v., but were more resistant when infected in a dermal site, although the later was only analyzed at a single early time point (Rousseau et al., 2001; Thalhofer et al., 2011).

\section{CONCLUSIONS}

Neutrophils have emerged as an important cell during Leishmania infection. Recent studies using fluorescent parasites, transgenic reporters mice, in vivo imaging and flow cytometry have confirmed neutrophils as the first cells recruited to and infected in the dermal site of inoculation (Peters et al., 2008, 2009; Thalhofer et al., 2011; Ribeiro-Gomes et al., 2012). 




FIGURE 1 | The immunomodulatory role of neutrophils at the site of Leishmania transmission by sand fly bite. Metacyclic promastigotes deposited in the skin by infected sand flies are taken up primarily by neutrophils that are rapidly recruited to the site of bite. Infected neutrophils recovered from the ear dermis have accelerated expression of apoptotic markers, with the majority dying in situ to release viable parasites that may be better adapted for intracellular survival and growth following their uptake by macrophages. The engulfment of infected and uninfected cells or apoptotic bodies by macrophages may inhibit the activation of infected macrophages in the inflammatory site. The initial infection of dermal DCs appears to take place primarily in the context of their capture of parasitized neutrophils. This encounter strongly reduces their priming capacity and consequently delays the immune response against the parasite until the acute neutrophilic response is resolved.

Elimination of the acute neutrophilic infiltrate enhanced host resistance to sand fly transmitted infection. Thus in the most relevant inflammatory setting initiated by the bite of an infected sand fly, in which the acute neutrophilic response is especially massive, localized and sustained, neutrophils can be shown to suppress immunity and promote the early infectious process. The possible mechanisms that might account for these effects are summarized in Figure 1.

The low efficacy of killed Leishmania vaccines against sand fly challenge may be directly related to the suppressor effects of neutrophils on dermal DCs. More generally, the effects of in vivo

\section{REFERENCES}

Afonso, L., Borges, V. M., Cruz, H., Ribeiro-Gomes, F. L., DosReis, G. A., Dutra, A. N., Clarencio, J., de Oliveira, C. I., Barral, A., BarralNetto, M., and Brodskyn, C. I. (2008). Interactions with apoptotic but not with necrotic neutrophils increase parasite burden in human macrophages infected with Leishmania amazonensis. J. Leukoc. Biol. 84, 389-396.
Aga, E., Katschinski, D. M., van Zandbergen, G., Laufs, H., Hansen, B., Muller, K., Solbach, W., and Laskay, T. (2002). Inhibition of the spontaneous apoptosis of neutrophil granulocytes by the intracellular parasite Leishmania major. J. Immunol. 169, 898-905.

al Tuwaijri, A. S., al Mofleh, I. A., and Mahmoud, A. A. (1990). Effect of Leishmania major on human polymorphonuclear leucocyte function

neutrophil depletion need to be more carefully interpreted with respect to the specificity of the antibodies used, the tissue site of challenge, and the timing of the treatments and analyzes that may lead to different conclusions depending on whether only the acute and/or subsequent recruitment to the inflammatory site has been eliminated.

\section{ACKNOWLEDGMENTS}

This work was supported in part by the Intramural Research Program of the National Institutes of Health, National Institute of Allergy and Infectious Diseases.

in vitro. J. Med. Microbiol. 32, 189-193.

Andrade, Z. A., Reed, S. G., Roters, S. B., and Sadigursky, M. (1984). Immunopathology of experimental cutaneous leishmaniasis. Am. J. Pathol. 114, 137-148.

Beil, W. J., Meinardus-Hager, G., Neugebauer, D. C., and Sorg, C. (1992). Differences in the onset of the inflammatory response to cutaneous leishmaniasis in resistant and susceptible mice. J. Leukoc. Biol. 52, 135-142.

Chang, K. P. (1981). Leishmanicidal mechanisms of human polymorphonuclear phagocytes. Am. J. Trop. Med. Hyg. 30, 322-333.

Charmoy, M., Auderset, F., Allenbach, C., and Tacchini-Cottier, F. (2010a). The prominent role of neutrophils during the initial phase of infection by Leishmania parasites. J. Biomed. Biotechnol. 2010, 719361. 
Charmoy, M., Brunner-Agten, S., Aebischer, D., Auderset, F., Launois, P., Milon, G., Proudfoot, A. E., and Tacchini-Cottier, F. (2010b). Neutrophil-derived CCL3 is essential for the rapid recruitment of dendritic cells to the site of Leishmania major inoculation in resistant mice. PLoS Pathog. 6:e1000755. doi: 10.1371/journal. ppat. 1000755

Charmoy, M., Megnekou, R., Allenbach, C., Zweifel, C., Perez, C., Monnat, K., Breton, M., Ronet, C., Launois, P., and Tacchini-Cottier, F. (2007). Leishmania major induces distinct neutrophil phenotypes in mice that are resistant or susceptible to infection. J. Leukoc. Biol. 82, 288-299.

Chen, L., Zhang, Z. H., Watanabe, T., Yamashita, T., Kobayakawa, T., Kaneko, A., Fujiwara, H., and Sendo, F. (2005). The involvement of neutrophils in the resistance to Leishmania major infection in susceptible but not in resistant mice. Parasitol. Int. 54, 109-118.

Daley, J. M., Thomay, A. A., Connolly, M. D., Reichner, J. S., and Albina, J. E. (2008). Use of Ly6G-specific monoclonal antibody to deplete neutrophils in mice. J. Leukoc. Biol. 83, 64-70.

Deane and Guimarges. (1938). Aspectos interessantes da fagocitose observados na leishmaniose visceral e na malaria. Mem. Inst. Oswaldo Cruz 33.

de Moura, T. R., Oliveira, F., Rodrigues, G. C., Carneiro, M. W., Fukutani, K. F., Novais, F. O., Miranda, J. C., Barral-Netto, M., Brodskyn, C., Barral, A., and de Oliveira, C. I. (2010). Immunity to Lutzomyia intermedia saliva modulates the inflammatory environment induced by Leishmania braziliensis. PLoS Negl. Trop. Dis. 4:e712. doi: 10.1371/journal.pntd.0000712

de Souza Carmo, E. V., Katz, S. and Barbieri, C. L. (2010). Neutrophils reduce the parasite burden in Leishmania (Leishmania) amazonensis-infected macrophages. PLoS One 5:e13815. doi: 10.1371/journal.pone.0013815

Filardy, A. A., Pires, D. R., Nunes, M. P., Takiya, C. M., Freire-deLima, C. G., Ribeiro-Gomes, F. L., and DosReis, G. A. (2010). Proinflammatory clearance of apoptotic neutrophils induces an IL12(low)IL-10(high) regulatory phenotype in macrophages. J. Immunol. 185, 2044-2050.

Fleming, T. J., Fleming, M. L., and Malek, T. R. (1993). Selective expression of Ly-6G on myeloid lineage cells in mouse bone marrow. RB6-8C5 mAb to granulocytedifferentiation antigen (Gr-1) detects members of the Ly- 6 family. J. Immunol. 151, 2399-2408.

Gabriel, C., McMaster, W. R., Girard, D., and Descoteaux, A. (2010). Leishmania donovani promastigotes evade the antimicrobial activity of neutrophil extracellular traps. J. Immunol. 185, 4319-4327.

Geissmann, F., Jung, S., and Littman, D. R. (2003). Blood monocytes consist of two principal subsets with distinct migratory properties. Immunity 19, 71-82.

Goncalves, R., Zhang, X., Cohen, H., Debrabant, A., and Mosser, D. M. (2011). Platelet activation attracts a subpopulation of effector monocytes to sites of Leishmania major infection. J. Exp. Med. 208, 1253-1265.

Gueirard, P., Laplante, A., Rondeau, C., Milon, G., and Desjardins, M (2008). Trafficking of Leishmania donovani promastigotes in nonlytic compartments in neutrophils enables the subsequent transfer of parasites to macrophages. Cell Microbiol. 10, 100-111.

Guimaraes-Costa, A. B., Nascimento, M. T., Froment, G. S., Soares, R. P., Morgado, F. N., Conceicao-Silva, F., and Saraiva, E. M. (2009) Leishmania amazonensis promastigotes induce and are killed by neutrophil extracellular traps. Proc. Natl. Acad. Sci. U.S.A. 106, 6748-6753.

Jacobs, T., Andra, J., Gaworski, I., Graefe, S., Mellenthin, K., Kromer, M., Halter, R., Borlak, J., and Clos, J. (2005). Complement C3 is required for the progression of cutaneous lesions and neutrophil attraction in Leishmania major infection. Med. Microbiol. Immunol. 194, 143-149.

Kim, J. V., and Dustin, M. L. (2006). Innate response to focal necrotic injury inside the blood-brain barrier. J. Immunol. 177, 5269-5277.

Kobayashi, S. D., and DeLeo, F. R. (2009). Role of neutrophils in innate immunity: a systems biology-level approach. Wiley Interdiscip. Rev. Syst. Biol. Med. 1, 309-333.

Kono, H., and Rock, K. L. (2008). How dying cells alert the immune system to danger. Nat. Rev. Immunol. 8, 279-289.

Kostka, S., Dinges, S., Griewank, K., Iwakura, Y., Udey, M. C., and von Stebut, E. (2009). IL-17 promotes progression of cutaneous leishmaniasis in susceptible mice. J. Immunol. 182, 3039-3046.

Kusmartsev, S. A., Li, Y., and Chen, S. H. (2000). Gr-1+ myeloid cells derived from tumor-bearing mice inhibit primary $\mathrm{T}$ cell activation induced through CD3/CD28 costimulation. J. Immunol. 165, 779-785.

Laskay, T., van Zandbergen, G., and Solbach, W. (2008). Neutrophil granulocytes as host cells and transport vehicles for intracellular pathogens: apoptosis as infectionpromoting factor. Immunobiology $213,183-191$.

Laufs, H., Muller, K., Fleischer, J., Reiling, N., Jahnke, N., Jensenius, J. C., Solbach, W., and Laskay, T. (2002). Intracellular survival of Leishmania major in neutrophil granulocytes after uptake in the absence of heat-labile serum factors. Infect. Immun. 70, 826-835.

Lima, G. M., Vallochi, A. L., Silva, U. R., Bevilacqua, E. M., Kiffer, M. M. and Abrahamsohn, I. A. (1998). The role of polymorphonuclear leukocytes in the resistance to cutaneous Leishmaniasis. Immunol. Lett. 64 145-151.

Matsuzaki, J., Tsuji, T., Chamoto, K., Takeshima, T., Sendo, F., and Nishimura, T. (2003). Successful elimination of memory-type CD8+ $\mathrm{T}$ cell subsets by the administration of anti-Gr-1 monoclonal antibody in vivo. Cell Immunol. 224, 98-105.

McDonald, B., Pittman, K., Menezes, G. B., Hirota, S. A., Slaba, I., Waterhouse, C. C., Beck, P. L., Muruve, D. A., and Kubes, P. (2010). Intravascular danger signals guide neutrophils to sites of sterile inflammation. Science 330, 362-366.

McFarlane, E., Perez, C., Charmoy, M., Allenbach, C., Carter, K. C., Alexander, J., and Tacchini-Cottier, F. (2008). Neutrophils contribute to development of a protective immune response during onset of infection with Leishmania donovani. Infect. Immun. 76, 532-541.

Mollinedo, F., Janssen, H., de la IglesiaVicente, J., Villa-Pulgarin, J. A., and Calafat, J. (2010). Selective fusion of azurophilic granules with Leishmania-containing phagosomes in human neutrophils. J. Biol. Chem. 285, 34528-34536.

Muller, K., van Zandbergen, G., Hansen, B., Laufs, H., Jahnke, N. Solbach, W., and Laskay, T. (2001). Chemokines, natural killer cells and granulocytes in the early course of Leishmania major infection in mice. Med. Microbiol. Immunol. 190, 73-76.

Nakano, H., Yanagita, M. and Gunn, M. D. (2001). $\mathrm{CD} 11 \mathrm{c}(+) \mathrm{B} 220(+) \mathrm{Gr}-1(+)$ cells in mouse lymph nodes and spleen display characteristics of plasmacytoid dendritic cells. J. Exp. Med. 194, 1171-1178.

Novais, F. O., Santiago, R. C., Bafica, A., Khouri, R., Afonso, L., Borges, V. M., Brodskyn, C., Barral-Netto, M., Barral, A., and de Oliveira, C. I. (2009). Neutrophils and macrophages cooperate in host resistance against Leishmania braziliensis infection. J. Immunol. 183, 8088-8098.

Pearson, R. D., and Steigbigel, R. T. (1981). Phagocytosis and killing of the protozoan Leishmania donovani by human polymorphonuclear leukocytes. J. Immunol. 127, 1438-1443.

Peters, N. C., Egen, J. G., Secundino, N., Debrabant, A., Kimblin, N., Kamhawi, S., Lawyer, P., Fay, M. P., Germain, R. N., and Sacks, D. (2008). In vivo imaging reveals an essential role for neutrophils in leishmaniasis transmitted by sand flies. Science 321 , 970-974.

Peters, N. C., Kimblin, N., Secundino, N., Kamhawi, S., Lawyer, P., and Sacks, D. L. (2009). Vector transmission of leishmania abrogates vaccine-induced protective immunity. PLoS Pathog. 5:e1000484. doi: 10.1371/journal.ppat.1000484

Pompeu, M. L., Freitas, L. A., Santos, M. L., Khouri, M., and BarralNetto, M. (1991). Granulocytes in the inflammatory process of $\mathrm{BALB} / \mathrm{C}$ mice infected by Leishmania amazonensis. A quantitative approach. Acta Trop. 48, 185-193.

Prates, D. B., Araujo-Santos, T., Luz, N. F., Andrade, B. B., FrancaCosta, J., Afonso, L., Clarencio, J., Miranda, J. C., Bozza, P. T., Dosreis, G. A., Brodskyn, C., Barral-Netto, M., Borges, V. M., and Barral, A (2011). Lutzomyia longipalpis saliva drives apoptosis and enhances parasite burden in neutrophils. J. Leukoc. Biol. 90, 575-582.

Remaley, A. T., Kuhns, D. B., Basford, R. E., Glew, R. H., and Kaplan, S. S. (1984). Leishmanial phosphatase blocks neutrophil O-2 production. J. Biol. Chem. 259, 11173-11175.

Ribeiro-Gomes, F. L., Moniz-de-Souza, M. C., Alexandre-Moreira, M. S., Dias, W. B., Lopes, M. F., Nunes, M. P., Lungarella, G., and DosReis. G. A. (2007). Neutrophils activate macrophages for intracellular killing of Leishmania major through recruitment of TLR4 by neutrophil elastase. J. Immunol. 179, 3988-3994.

Ribeiro-Gomes, F. L., Otero, A. C., Gomes, N. A., Moniz-DeSouza, M. C., Cysne-Finkelstein, L., Arnholdt, A. C., Calich, V. 
L., Coutinho, S. G., Lopes, M. F., and DosReis, G. A. (2004). Macrophage interactions with neutrophils regulate Leishmania major infection. J. Immunol. 172, 4454-4462.

Ribeiro-Gomes, F., Peters, N. C., Debrabant, A., and Sacks, D. A. (2012). Efficient capture of infected neutrophils by dendritic cells in the skin inhibits the early anti-Leishmania response. PloS Pathog. 8:e1002536. doi: 10.1371/journal.ppat.1002536

Rousseau, D., Demartino, S., Ferrua, B., Michiels, J. F., Anjuere, F., Fragaki, K., Fichoux, Y. L., and Kubar, J. (2001). In vivo involvement of polymorphonuclear neutrophils in Leishmania infantum infection. BMC Microbiol. 1, 17.

Sacks, D. L. (1992). The structure and function of the surface lipophosphoglycan on different developmental stages of Leishmania promastigotes. Infect. Agents Dis. 1, 200-206.

Segal, A. W. (2005). How neutrophils kill microbes. Annu. Rev. Immunol. 23, 197-223.

Smelt, S. C., Cotterell, S. E., Engwerda, C. R., and Kaye, P. M. (2000).
B cell-deficient mice are highly resistant to Leishmania donovani infection, but develop neutrophilmediated tissue pathology. J. Immunol. 164, 3681-3688.

Tacchini-Cottier, F., Zweifel, C., Belkaid, Y., Mukankundiye, C., Vasei, M., Launois, P., Milon, G., and Louis, J. A. (2000). An immunomodulatory function for neutrophils during the induction of a CD4+ Th2 response in BALB/c mice infected with Leishmania major. J. Immunol. 165, 2628-2636.

Teixeira, C. R., Teixeira, M. J., Gomes, R. B., Santos, C. S., Andrade, B. B., Raffaele-Netto, I., Silva, J. S., Guglielmotti, A., Miranda, J. C., Barral, A., Brodskyn, C., and Barral-Netto, M. (2005). Saliva from Lutzomyia longipalpis induces CC chemokine ligand 2/monocyte chemoattractant protein-1 expression and macrophage recruitment. J. Immunol. 175, 8346-8353.

Tepper, R. I., Coffman, R. L., and Leder, P. (1992). An eosinophil-dependent mechanism for the antitumor effect of interleukin-4. Science 257, 548-551.

Thalhofer, C. J., Chen, Y., Sudan, B., Love-Homan, L., and Wilson, M.
E. (2011). Leukocytes infiltrate the skin and draining lymph nodes in response to the protozoan Leishmania infantum chagasi. Infect. Immun. 79, 108-117.

van Zandbergen, G., Hermann, N., Laufs, H., Solbach, W., and Laskay. T. (2002). Leishmania promastigotes release a granulocyte chemotactic factor and induce interleukin-8 release but inhibit gamma interferon-inducible protein 10 production by neutrophil granulocytes. Infect. Immun. 70 , 4177-4184.

van Zandbergen, G., Klinger, M., Mueller, A., Dannenberg, S., Gebert, A., Solbach, W., and Laskay, T. (2004). Cutting edge: neutrophil granulocyte serves as a vector for Leishmania entry into macrophages. J. Immunol. 173, 6521-6525.

Vento, S., and Cainelli, F. (2003). Infections in patients with cancer undergoing chemotherapy: aetiology, prevention, and treatment. Lancet Oncol. 4, 595-604.

Wenzel, A., and van Zandbergen, G. (2009). Lipoxin A4 receptor dependent leishmania infection. Autoimmunity 42, 331-333.
Wilson, M. E., Innes, D. J., Sousa, A. D., and Pearson, R. D. (1987). Early histopathology of experimental infection with Leishmania donovani in hamsters. J. Parasitol. 73, 55-63.

Conflict of Interest Statement: The authors declare that the research was conducted in the absence of any commercial or financial relationships that could be construed as a potential conflict of interest.

Received: 16 February 2012; paper pending published: 18 March 2012; accepted: 16 April 2012; published online: 04 May 2012.

Citation: Ribeiro-Gomes FL and Sacks D (2012) The influence of early neutrophilLeishmania interactions on the host immune response to infection. Front. Cell. Inf. Microbio. 2:59. doi: 10.3389/ fcimb.2012.00059

Copyright (c) 2012 Ribeiro-Gomes and Sacks. This is an open-access article distributed under the terms of the Creative Commons Attribution Non Commercial License, which permits non-commercial use, distribution, and reproduction in other forums, provided the original authors and source are credited. 